\title{
NEW CHROMOSOME COUNTS OF ASIAN COSTACEAE AND INITIAL INSIGHTS INTO THE GENOME EVOLUTION OF THE FAMILY
}

\author{
P. H. van Caspel ${ }^{1 *}$, A. D. Poulsen ${ }^{1} \&$ M. Möller ${ }^{1}$
}

\begin{abstract}
Chromosome counts were obtained from six species of Costaceae from Asia. Our count of $2 n$ $=18$ for Cheilocostus speciosus confirms previous counts, and the other five counts have been made for the first time (Cheilocostus borneensis, Cheilocostus globosus, Cheilocostus sopuensis, Costus muluensis and Paracostus sp.). These chromosome counts reveal two somatic numbers, $2 n=18$ and $2 n=36$, of which the former is a new diploid number for the genus Paracostus. A comprehensive review of existing counts was conducted through literature and database searches. Mapping of these on a published comprehensive phylogenetic tree suggests that the diploid count of $2 n=18$ is probably ancestral in the Costaceae, with repeated parallel evolution of tetraploidy and one case of octoploidy. The existence of triploid counts in several lineages harbouring polyploids suggests that diploids and tetraploids may exist in close proximity, and that crosses or meiotic irregularities may lead to triploid genotypes occurring frequently.
\end{abstract}

Keywords. Borneo, Cheilocostus, Costus, cytology, Hellenia, Paracostus.

Received 4 May 2020 Accepted 14 September 2020 Published 10 March 2021

\section{Introduction}

Relatively few cytological studies have focused on the tropical plant family Costaceae Nakai. So far, the majority of chromosome numbers obtained are of Neotropical members of the family, which show a predominant somatic number of $2 n=18$ (Mahanty, 1970; Maas, 1972), and only four Asian species of the family have been counted (Table 1). All Asian Costaceae share the somatic number of $2 n=18$, except for Cheilocostus speciosus (J.Koenig) C.D.Specht. This species shows counts varying between $2 n=13$ and $2 n=72$, with the majority being $2 n=36$ (see Table 1 ). Most counts for the family are $2 n=18$, which may suggest that $x=9$ is the basic number in Costaceae, and $2 n=18$ the ancestral state, but this has never been investigated for the family, only proposed for the genus Costus L. (Maas, 1972). Regarding Paracostus C.D.Specht, only one count $(2 n=36)$ is known for the African P. englerianus (K.Schum.) C.D.Specht. This count has not been confirmed, and more studies are needed to investigate whether this is a tetraploid species or represents a tetraploid population (Mahanty, 1970).

In 2006, Specht published a generic recircumscription of Costaceae resulting in the restriction of Costus to Africa and the Neotropics. The Asian species formerly in Costus were instead placed in Cheilocostus C.D.Specht and Paracostus, although combinations in these genera were not made for all Asian species, for example Costus tonkinensis Gagnep.

${ }^{1}$ Royal Botanic Garden Edinburgh, 20A Inverleith Row, Edinburgh EH3 5LR, Scotland, UK.

* Corresponding author. E-mail: phcaspel@protonmail.com. 


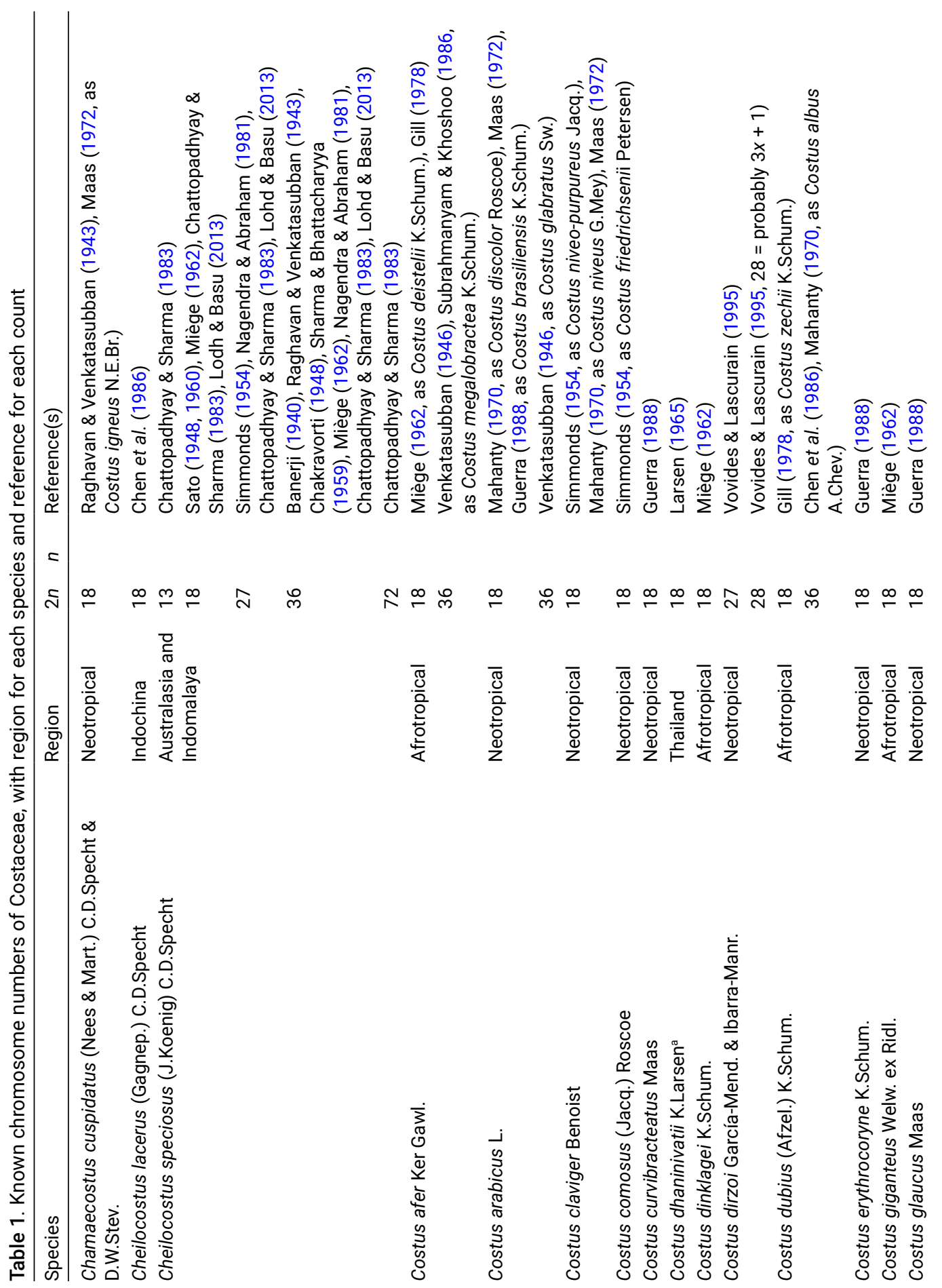




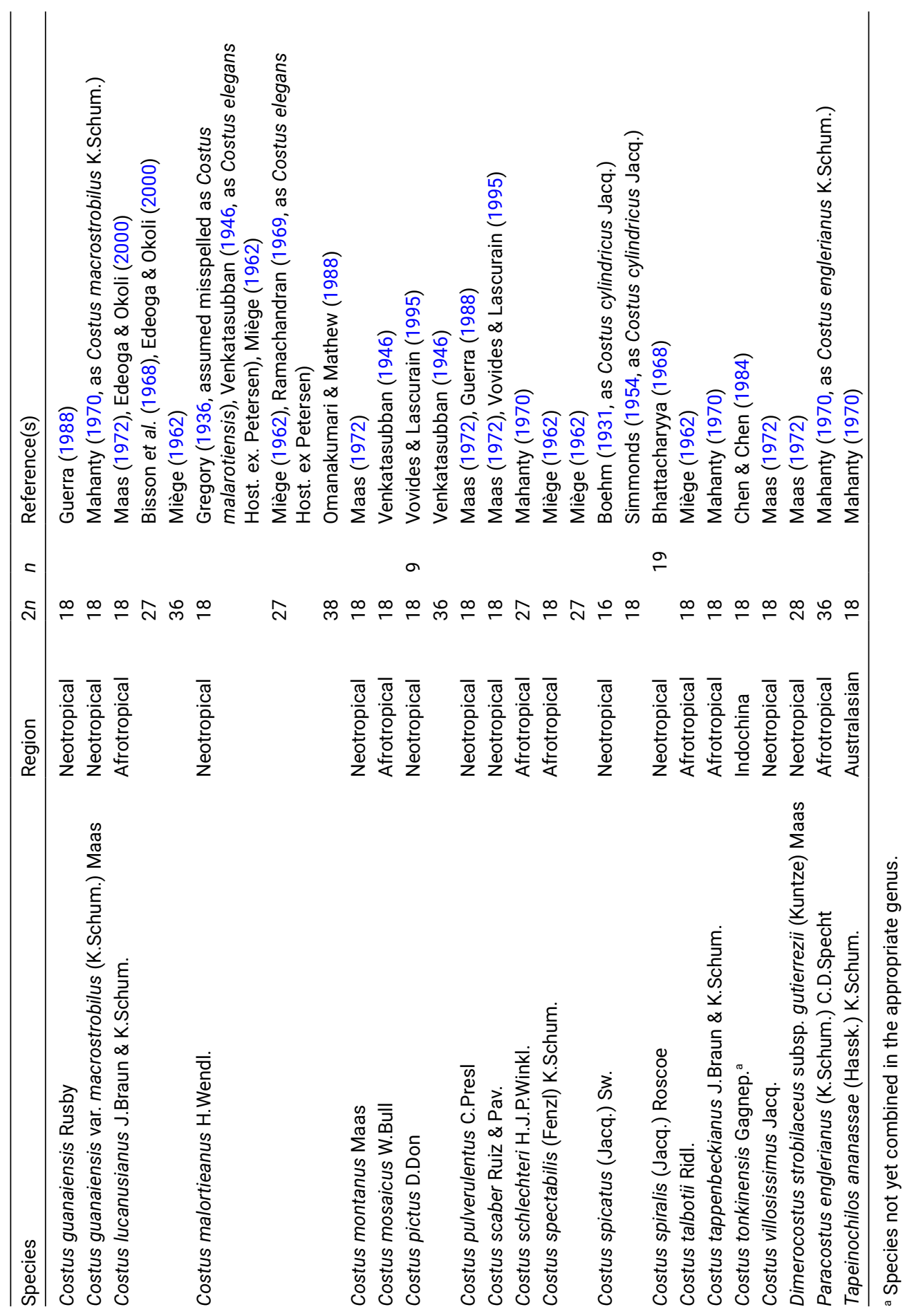


(Specht \& Stevenson, 2006). Choosing to ignore this recircumscription, Meekiong et al. (2006) subsequently published Costus muluensis Meekiong, Ipor \& Tawan in Costus subg. Paracostus K.Schum. A paper is in preparation to make the necessary combination in Paracostus.

The name Cheilocostus is superfluous because an older name is available: Hellenia Retz. This name, however, is confusing, because Hellenia Willd. nom. illeg. has been used widely for species now placed in Alpinia Roxb. in Zingiberaceae, the sister family to Costaceae. A proposal to conserve the name Cheilocostus, or at least move away from the confusing Hellenia, has therefore been initiated (Leong-Škorničková \& Šída, 2016). Because a decision has yet to be made, we adhere in this publication to the use of the generic name Cheilocostus so as not to confuse the situation further or to establish the use of Hellenia.

Chromosome counts in members of the Costaceae date back to 1931, when Boehm, relying on pollen mother cells, established a count of $x=8$ for Costus spicatus (Jacq.) Sw. This number has been doubted by Mahanty $(1970$, p. 37), because of a later count in this species of $2 n=18$ (Simmonds, 1954). Early work relied on the paraffin-sectioning method, which may obscure chromosome details (Gregory, 1936; Raghavan \& Venkatasubban, 1943; Venkatasubban, 1946; Sato, 1948, 1960; Mahanty, 1970; Maas, 1972). More recent work has used the root-tip squash method (e.g. Ramachandran, 1969; Mahanty, 1970; Subrahmanyam \& Khoshoo, 1986). These later authors also employed Feulgen staining to overcome the problem, previously noted by Mahanty (1970), of stainability of Zingiberales mitotic chromosomes.

Although some doubtful counts may be attributable to methodological obstacles, some odd counts appear repeatedly, with $2 n=3 x=27$ for some species (e.g. Mahanty, 1970; Subrahmanyam \& Khoshoo, 1986; Lohd \& Basu, 2013) (see Table 1). These may represent triploids of crosses between diploid and tetraploid genotypes. Polyploids can basically arise in two forms: as autopolyploids possessing sets of identical chromosomes originating from meiotic replication errors and/or fusion of unreduced gametes forming multivalents at meiosis; and as allopolyploids having two different sets of chromosomes, each haploid set from a different species, with subsequent genome duplication forming bivalents at meiosis (Stebbins, 1971; Levin, 2002). Autopolyploidy is a common feature in vegetatively propagating plants (Mahanty, 1970; Meyers \& Levin, 2006; Lohd \& Basu, 2013).

The ecological advantage of polyploidy would be the ability to carry more than two alleles (fixed in allopolyploids) over diploids, which might result in superior genotypes (e.g. Otto, 2007; Alix et al., 2017). Whole-genome duplication events (autopolyploidy) have occurred repeatedly throughout the evolution of the angiosperms, including several times in monocots (Weiss-Schneeweiss, 2013; Landis et al., 2018), and it is estimated that about $70 \%$ of angiosperms have experienced increases in ploidy level (Meyers \& Levin, 2006), largely because polyploidy is irreversible (Stebbins, 1971; Grant, 1981). Although diploid and polyploid counts in the family Costaceae have been published, genome evolution within the family is as yet unstudied. 
We aimed in the present study to add to the scant counts of Asian members of the Costaceae and to fill gaps in our knowledge of this group, with a focus on Bornean representatives. At the same time, we hoped to supplement the existing counts in the family and study the evolution of their genomes, elucidating the basic number and ploidy level in a phylogenetic context in the family.

\section{Materials and methods}

The living collections at the Royal Botanic Garden Edinburgh (RBGE) enabled us to include the following Asian species in the present study: Cheilocostus borneensis A.D.Poulsen, $C$. globosus (Blume) C.D.Specht, C. sopuensis (Maas \& H.Maas) C.D.Specht, C. speciosus, Costus muluensis and a species of Paracostus that is probably undescribed (Table 2). Eight accessions of the six species in the three genera were sampled, with two samples each for Cheilocostus globosus and C. muluensis. To increase the chances of cytological success, stem cuttings were taken and cultivated to produce fresh, actively dividing roots, which were harvested about 6 weeks after the cuttings were taken.

The cytological methods followed Jong's (1997) Feulgen squash technique, with slight alterations. Briefly, root tips were pretreated in either 8-hydroxyquinoline or paradichlorobenzene in the dark for $5 \mathrm{~h}$ at room temperature. The roots were fixed in Farmer's fluid (3:1, ethanol:glacial acetic acid) and hydrolysed in $5 \mathrm{M}$ hydrochloric acid for $30 \mathrm{~min}$. They were then stained in freshly prepared Feulgen reagent (Fox, 1969) and placed in the dark for up to $2 \mathrm{~h}$. Softening of the roots was achieved by immersion in a 1:1 enzyme mixture of $4 \%$ pectinase and $4 \%$ cellulase at $36^{\circ} \mathrm{C}$ for $30 \mathrm{~min}$. Root-tip meristems were then squashed in $0.05 \%$ acetocarmine counterstain to reduce fading of the Feulgen stain over time in permanent slides.

Permanent slides were prepared using a vapour exchange method (Bradley, 1948; Jong, 1997). Images were captured using AxioVision Rel. v. 4.7 and an AxioCam MRc 5 camera mounted on an AxioPhot brightfield microscope (all Zeiss, Welwyn Garden City, UK). Root-tip squash preparations were repeated until at least two confirmatory counts had been obtained (see Table 2). Several images were recorded, but only one per species is shown in this paper.

The initial root harvest of Cheilocostus globosus did not lead to satisfactory preparations. A second root harvest was carried out and given a slightly altered pretreatment: roots were placed in 8-hydroxyquinoline at room temperature for $6 \mathrm{~h}$ instead of $5 \mathrm{~h}$.

To facilitate a discussion of the genome evolution of Costaceae, the phylogenetic tree of Specht (2006) was used to plot the chromosome numbers established for the family in this study, alongside counts from previous studies. The species identities of the accessions used for the counts were updated following the currently accepted synonymy. Furthermore, we believe Specht (2006) made a misidentification when including a sample of Paracostus from Borneo as P. paradoxus (K.Schum.) C.D.Specht. In the modified tree (see Figure 2), 


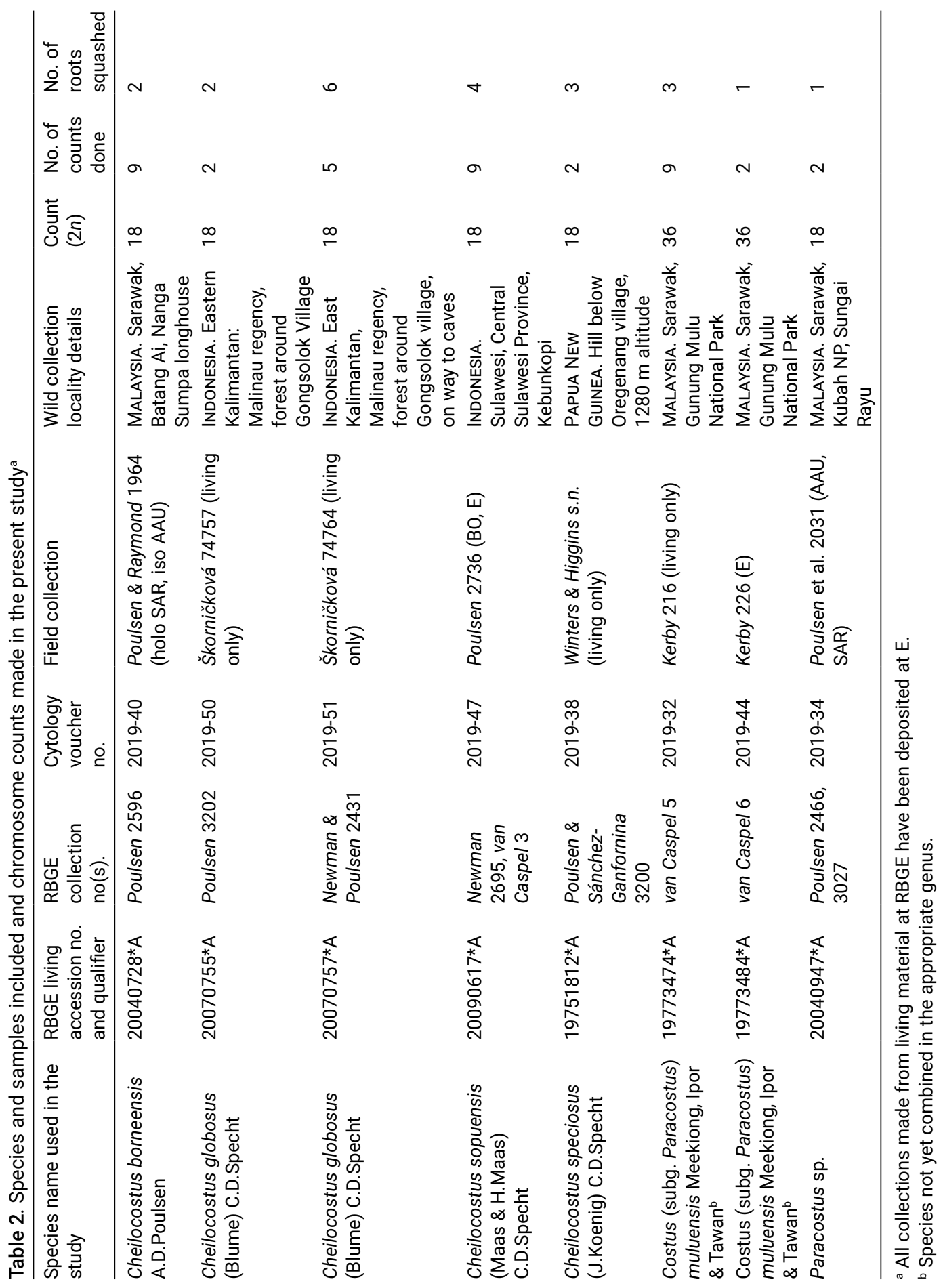


we have therefore labelled the branch as Paracostus spp. to represent Paracostus sp. and Costus muluensis, belonging to subgenus Paracostus, as explained above.

\section{Results and discussion}

In the present study, new counts of five species of Asian Costaceae were obtained and the count of Cheilocostus speciosus was confirmed. For two of the species, two accessions were examined and gave identical counts. One of the new counts is for an undescribed species of Paracostus (see Table 2, Figure 1). The chromosome counts revealed two somatic numbers among the samples, namely $2 n=18$ and $2 n=36$; the former is a new number in the genus Paracostus, the only other count available so far being $2 n=36$ in $P$. englerianus (Mahanty, 1970).

Most root-tip cell preparations showed chromosomes in prometaphase, so they were difficult to measure (see Figure 1). The length range of the metaphase chromosomes of Costus muluensis was between 1.2 and $3.5 \mu \mathrm{m}$, and for Cheilocostus sopuensis between 0.9 and $3.1 \mu \mathrm{m}$. The variation may be influenced by the condensation level of the chromosomes in the preparations, although Mahanty (1970) gives a length range for Costus guanaiensis var. macrostrobilus (K.Schum.) Maas (as C. macrostrobilus K.Schum.) of 2.3-3.7 $\mu \mathrm{m}$, which is well within the ranges of the species studied here. Similar ranges were reported by Subrahmanyam \& Khoshoo (1986) for Costus malortieanus H.Wendl. (1.3-2.5 $\mu \mathrm{m}, 2 x$ ) and $C$. afer Ker Gawl. (as C. megalobractea K.Schum.; 1.4-3.5 $\mu \mathrm{m}, 4 x$ ). In Cheilocostus, similar ranges were reported by Lodh \& Basu (2013) for $C$. speciosus: $1.47-3.27 \mu \mathrm{m}(2 x)$ to $1.60-4.37 \mu \mathrm{m}(4 x)$. The authors of these studies commented on the uniformly gradual series of longest to shortest chromosome, similar to the findings presented here.

Without exception, in the present study chromosome numbers were found to be consistent with a basic number of $x=9$. The numbers found in Cheilocostus globosus $\left(20070757{ }^{\star} A, 2 n=18\right)$ and $C$. speciosus $\left(19751812^{\star} A, 2 n=18\right)$ matched that of their presumed congeners in Cheilocostus: Costus tonkinensis (placed within the Globosus complex; Maas, 1979) and Cheilocostus lacerus (Gagnep.) C.D.Specht (each $2 n=18$ ). The Malaysian Costus muluensis $\left(19773484^{\star} \mathrm{A}\right)$, still to be combined in Paracostus, had a chromosome count of $2 n=36$, equal to its African relative Paracostus englerianus. Paracostus sp. from Borneo (20040947*A) and Cheilocostus sopuensis $\left(20090617{ }^{\star} A\right)$ both had $2 n=18$, a number widespread in the family. Across all counts in the family, only Dimerocostus Kuntze deviated from the basic number of $x=9$ and has previously been counted with $x=14$ chromosomes (see Table 1) (Maas, 1972).

Infraspecific variation in chromosome numbers has previously been attributed to differences at the population level in several species (see Table 1). For instance, in Cheilocostus speciosus, a range of ploidy counts varying from diploid to octoploid including a triploid were determined for different populations (see Table 1), although no explanation was given for the odd count of $2 n=13$ by Chattopadhyay \& Sharma (1983). Others, however, 


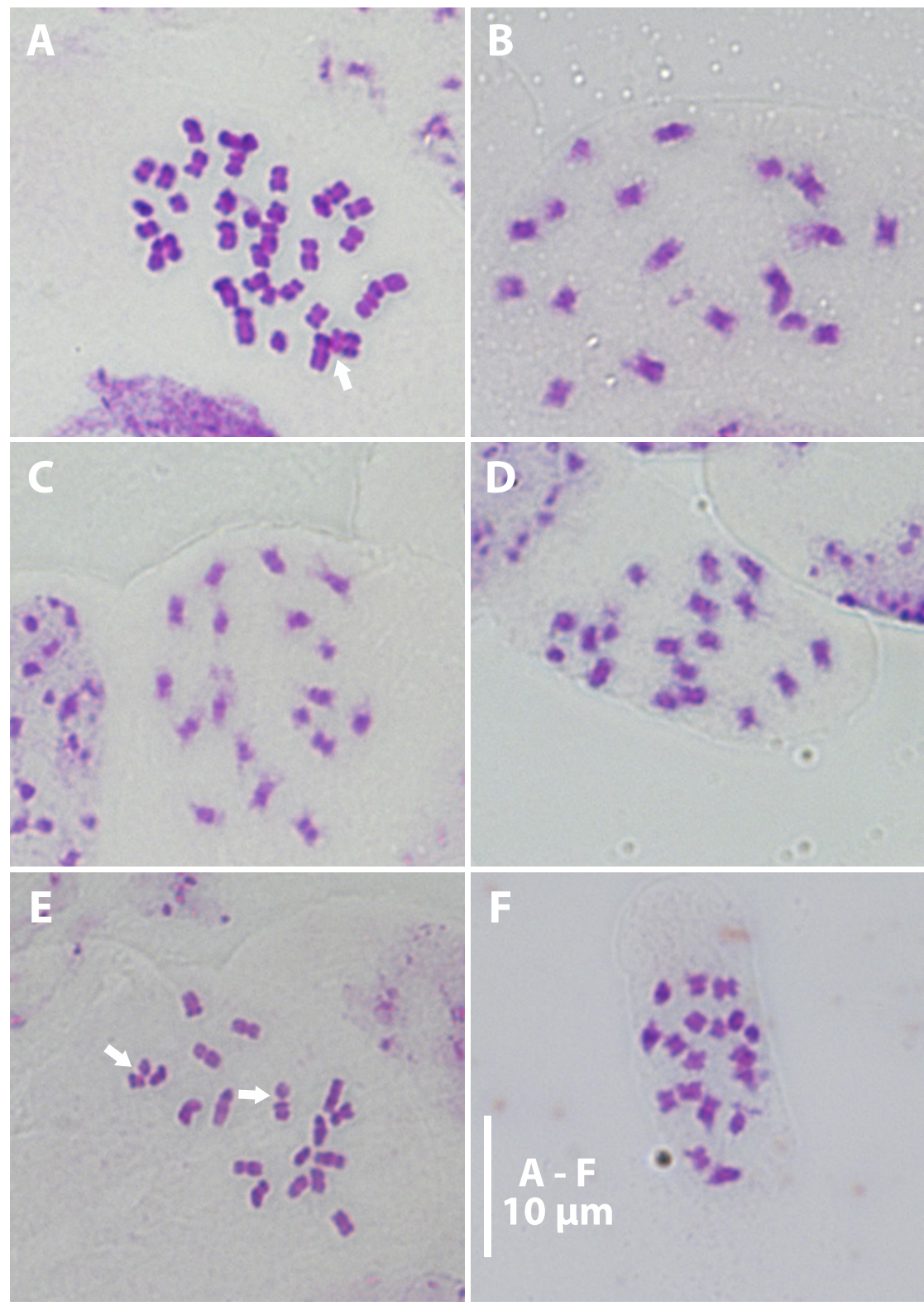

Figure 1. Root-tip chromosome squash preparations for members of Costaceae (garden accession numbers are in parentheses). A, Metaphase of Costus muluensis with $2 n=36(19773474 * A)$; the arrow indicates a squashed chromosome. B, Prometaphase of Paracostus sp. with $2 n=18\left(20040947^{\star} A\right)$. C, Prometaphase of Cheilocostus borneensis with $2 n=18\left(20040728^{*} A\right)$. D, Protometaphase of Cheilocostus globosus with $2 n=18\left(20070757^{\star} \mathrm{A}\right)$. E, Metaphase of Cheilocostus sopuensis with $2 n=18$ $\left(20090617^{\star} A\right)$; the arrows indicate possible secondary constrictions. F, Late prometaphase of Cheilocostus speciosus with $2 n=18\left(19751812^{\star} \mathrm{A}\right)$. All images are at the same scale (scale bar, $\left.10 \mu \mathrm{m}\right)$. 
considered Cheilocostus speciosus to represent a species complex, with several varieties currently placed within the species, based on the similar vegetative and floral morphology (Specht \& Stevenson, 2006; Harrington \& Zich, 2012). In fact, new species closely related to Cheilocostus speciosus have been described recently (Harrington \& Zich, 2012; Kumar et al., 2016), indicating that this species may be split up. Future molecular studies may reveal that some of the varieties currently described could be recognised at species level or sunk, depending on the results. Some of the cytological differences may be attributable to different species or varieties, or the fact that morphological differences between taxa are an expression of their different ploidy levels (e.g. Mahanty, 1970; Lohd \& Basu, 2013; WeissSchneeweiss, 2013; Kolář et al., 2017).

The existence of infraspecific ploidy level variation may indicate autopolyploidy as the mechanism for the increase in chromosome numbers, as suggested by Mahanty (1970) and Lohd \& Basu (2013). Such an autopolyploid scenario has been proposed for Costus Iucanusianus J.Braun \& K.Schum., probably through the formation of polyploids from unreduced gametes (Edeoga \& Okoli, 2000). Autopolyploidy would result in the formation of multivalents during meiosis, but in several independent studies of Costus speciosus, summarised by Subrahmanyam \& Khoshoo (1986, p. 739, and references therein), only bivalents were found in triploid and tetraploid plants. This may suggest that allopolyploidy, that is, hybridisation between different species followed by genome duplication, is the source of the polyploids (Lohd \& Basu, 2013). An alternative explanation could be diploidisation of autopolyploid plants, whereby duplicated genes are randomly lost over time until only two homologous genomes exist (e.g. Gatt et al., 1998; Dodsworth et al., 2016). Clearly, more work is required to understand the origin and nature of polyploidy in Costaceae.

Although we refrain from a formal character optimisation here, due to missing chromosome counts for a range of species included in the phylogenetic tree, some preliminary inferences can be made on genome evolution in the family. The diploid number of $2 n=18$ occurs across the phylogenetic tree of Costaceae (Figure 2), except for the clade on the basal-most lineage, and is probably the ancestral state in the family. To test this hypothesis, more counts are required in the Dimerocostus and Chamaecostus C.D.Specht \& D.W.Stev. clades. The only representative of the former cytologically investigated is Dimerocostus strobilaceus subsp. gutierrezii (Kuntze) Maas, which resulted in a unique count of $2 n=28$ (see Figure 2) (Maas, 1972). At present, it is unclear whether this is an autapomorphy of the species or a characteristic of the genus or the clade, or a miscount of a triploid, as observed in several other species, due to the sectioning method used by Maas (1972).

Tetraploids with $2 n=4 x=36$ appear scattered in several different places across the family phylogeny and have very probably evolved independently from each other. This is supported by the fact that in four out of six instances of tetraploidisation, diploids were also found (see Figure 2). The case of Paracostus is unclear, because the branch leading to Paracostus spp. includes data obtained from two different species (see above; see Figure 2). 


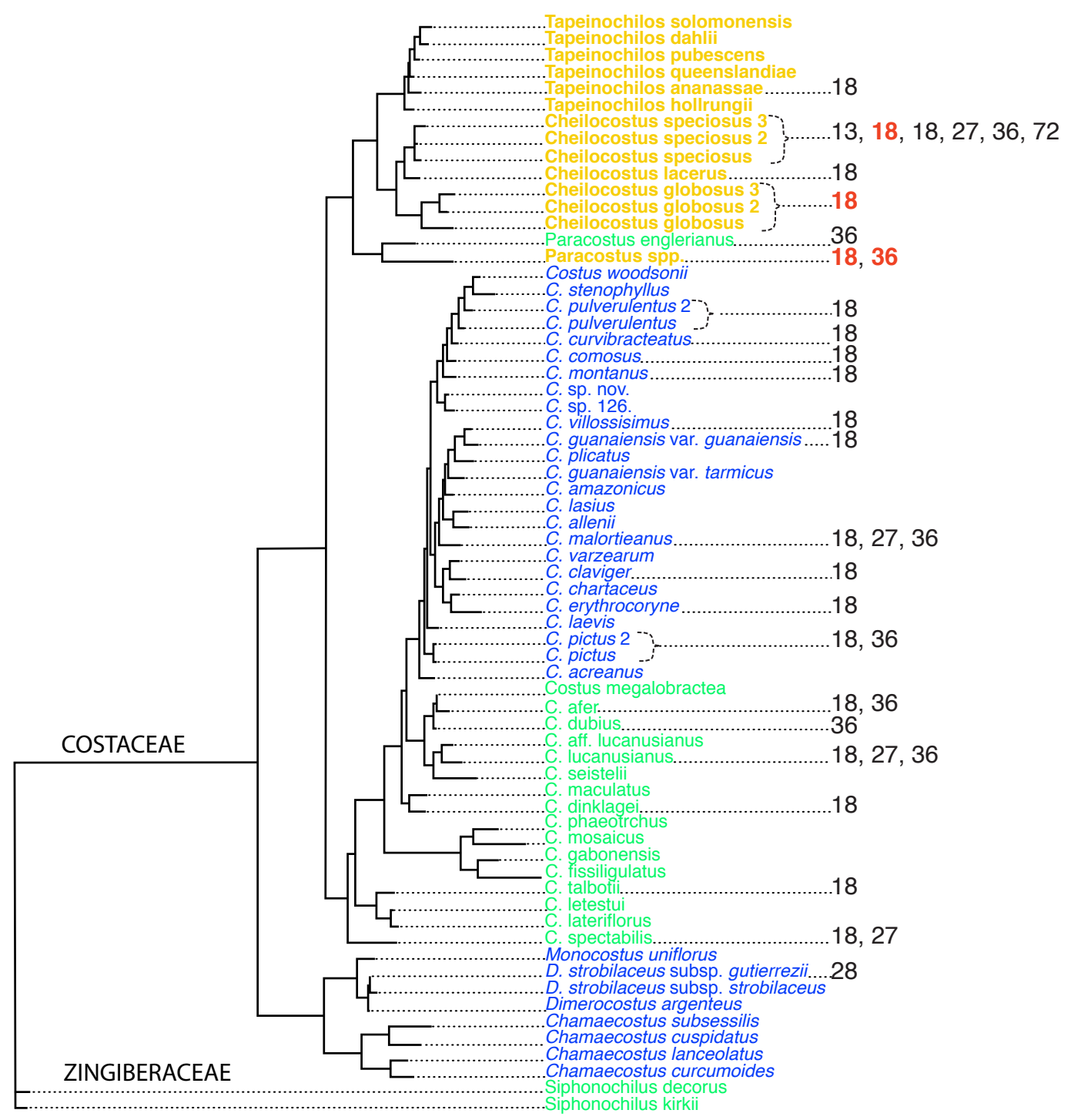

Figure 2. Phylogenetic tree of Costaceae, modified from Specht (2006), with chromosome numbers (2n) from previous studies in black (see Table 1 for references) and counts made in the present study in red. African species are in green roman text, Neotropical in blue italic text and Asian in yellow bold text. In the work of Specht, the Bornean Paracostus sample included was identified as $P$. paradoxus, which we believe is a misidentification; the branch has therefore been labelled Paracostus spp. and represents the undescribed Paracostus $(2 n=18)$ and Costus (subg. Paracostus) muluensis $(2 n=36)$. 
It is also interesting to note that in three out of seven polyploidisation events, triploids with $2 n=3 x=27$ were found (see Figure 2), suggesting crosses between diploid and tetraploid forms. Triploids or aneuploids also occur in many Zingiberaceae Martinov, such as Curcuma L. (Leong-Škorničková et al., 2007). Families in the order Zingiberales may be prone to meiotic errors and unbalanced karyotypes, and the persistence of sterile triploids may result from their rhizome-forming vegetative reproductive strategy. This is an area in which further studies are necessary.

In summary, the chromosome numbers of the Asian Costaceae studied here $(2 n=2 x$ $=18$ and $2 n=4 x=36$ ) are in line with those observed in other members of the family and share a common basic number of $x=9$. In phylogenetic terms, diploidy seems to have been ancestral, and polyploids seem to have arisen on several occasions independently. The mechanisms by which the polyploids arose may perhaps be different; evidence of both allopolyploidy and autopolyploidy exists, and no single mechanism may exist for Costaceae. The vegetative reproduction of the plants aids the persistence of odd polyploids and may be one facet of the scenario. To fully understand the situation, each case would require detailed studies, including meiotic and molecular studies. Our new counts presented here fill gaps in our knowledge but more work is needed.

\section{Acknowledgements}

The authors would like to extend their gratitude to Stephen Willis, for taking great care in growing the stem cuttings and keeping the ginger collection at RBGE in great shape; the Sibbald Trust, for financial support for the write-up of this study; and of course our RBGE colleagues Edeline Gagnon and Thibauld Michel, who have provided moral support. The Royal Botanic Garden Edinburgh is supported by the Scottish Government's Rural and Environmental Science and Analytical Services Division.

\section{References}

Alix K, Gérard PR, Schwarzacher T, Heslop-Harrison JS. 2017. Polyploidy and interspecific hybridization: partners for adaptation, speciation and evolution in plants. Annals of Botany. 120(2):183-194.

Banerji IA. 1940. A contribution to the life history of Costus speciosus Smith. Journal of the Indian Botanical Society. 19:181-196.

Bhattacharyya R. 1968. Systematic status of the family Zingiberaceae. Nucleus, Supplement. 39:41.

Bisson S, Guillemet S, Hamel J-L. 1968. Contribution à l'ètude caryo-taxonomique des Scitaminées. Mémoires du Muséum National d'Histoire Naturelle. 18:59-133.

Boehm K. 1931. Embryologische Untersuchungen an Zingiberaceen. Planta. 14(2):411-440.

Bradley MV. 1948. A method for making aceto-carmine squashes permanent without removal of the cover slip. Stain Technology. 23(1):41-44.

Chakravorti AK. 1948. Multiplication of chromosome numbers in relation to speciation of Zingiberaceae. Science and Culture. 14:137-140. 
Chattopadhyay S, Sharma AK. 1983. Genetic diversity in Costus speciosus (Koen.) Sm. Cytologia. 48(1):209-214.

Chen Z, Chen S. 1984. A report on chromosome numbers of Chinese Zingiberaceae. Guihaia. 4:13-18.

Chen Z-Y, Chen S-J, Huang S-F. 1986. A report on chromosome numbers on Chinese Zingiberaceae (3). Acta Botanica Austro Sinica. 3:57-61.

Dodsworth S, Chase MW, Leitch AR. 2016. Is post-polyploidization diploidization the key to the evolutionary success of angiosperms? Botanical Journal of the Linnean Society. 180(1):1-5.

Edeoga HO, Okoli BE. 2000. Chromosome numbers of Costus lucanusianus (Costaceae) in Nigeria. Folia Geobotanica. 35(3):35-318.

Fox DP. 1969. Some characteristics of the cold hydrolysis technique for staining plant tissue by Feulgen reaction. Journal of Histochemistry and Cytochemistry. 17(4):266-272.

Gatt M, Ding H, Hammett K, Murray B. 1998. Polyploidy and evolution in wild and cultivated Dahlia species. Annals of Botany. 81(5):647-656.

Gill LS. 1978. Chromosome numbers of angiosperms in Tanzania: II. Adansonia. 18:19-24.

Grant V. 1981. Plant Speciation, 2nd edition. New York: Columbia University Press.

Gregory PJ. 1936. The floral morphology and cytology of Elettaria cardamomum Maton. Journal of the Linnean Society, Botany. 50(334):363-391.

Guerra MS. 1988. Characterization of different types of condensed chromatin in Costus (Zingiberaceae). Plant Systematics and Evolution. 158:107-115.

Harrington M, Zich F. 2012. A new combination in Australian Costaceae. Telopea. 14:9-10.

Jong K. 1997. Laboratory Manual of Plant Cytological Techniques. Edinburgh: Royal Botanic Garden Edinburgh.

Kolář F, Čertner M, Suda J, Schönswetter P, Husband BC. 2017. Mixed-ploidy species: progress and opportunities in polyploid research. Trends in Plant Science. 22(12):1041-1055.

Kumar R, Singh SK, Sinha BK, Sharma S. 2016. Description of two new species of Hellenia (Costaceae) from North-east India. Keanean Journal of Science. 5:3-8.

Landis JB, Soltis DE, Li Z, Marx HE, Barker MS, Tank DC, Soltis PS. 2018. Impact of whole-genome duplication events on diversification rates in angiosperms. American Journal of Botany. 105(3):348-363.

Larsen K. 1965. Costus dhaninivatii, a new species from S.E. Thailand. Southeast Asian Studies. 149-152.

Leong-Škorničková J, Šída O. 2016. Proposal to conserve the name Cheilocostus against Hellenia and Tsiana (Costaceae). Taxon. 65(6):1426-1427.

Leong-Škorničková J, Šída O, Jarolímoá V, Sabu M, Fér T, Trávnícek P, Suda J. 2007. Chromosome numbers and genome size variation in Indian species of Curcuma (Zingiberaceae). Annals of Botany. 100(3):505-526.

Levin DA. 2002. The Role of Chromosomal Change in Plant Evolution. New York: Oxford University Press.

Lodh D, Basu S. 2013. Karyomorphological analysis and cytotypic diversity in natural populations of Costus speciosus Koen. ex Retz. Nucleus. 56(3):155-162. 
Maas PJM. 1972. Costoideae (Zingiberaceae). Flora Neotropica, monograph 8. New York: Hafner. Maas PJM. 1979. Notes on Asiatic and Australian Costoideae (Zingiberaceae). Blumea. 25(2):543-549.

Mahanty HK. 1970. A cytological study of the Zingiberales with special reference to their taxonomy. Cytologia. 35(1):13-49.

Meekiong K, Ipor IB, Tawan CS, Muliati M. 2006. Three new species from Sarawak, and notes on the cauliferous Costus Group (Costaceae, Subgenus Paracostus). Folia Malaysiana. 7(1-2):55-72.

Meyers LA, Levin DA. 2006. On the abundance of polyploids in flowering plants. Evolution. 60(6):1198-1206.

Miège J. 1962. Quatrieme liste de nombres chromosomiques d'especes d'Afrique Occidentale. Revue de Cytologie et de Biologie Végétales. 24:149-164.

Nagendra P, Abraham PZ. 1981. Polyploidy and speciation in Costus speciosus (Koen.) Sm. Current Science. 50:26-28.

Omanakumari N, Mathew PM. 1988. Karyomorphological studies on two species of Costus L. New Botanist: An International Quarterly Journal of Plant Science Research. 15:33-37.

Otto SP. 2007. The evolutionary consequences of polyploidy. Cell. 131(3):452-462.

Raghavan TS, Venkatasubban KR. 1943. Cytological studies in the family Zingiberaceae with special reference to chromosome numbers and cyto-taxonomy. Proceedings of the Indian Academy of Sciences, Section B. 17(4):118-132.

Ramachandran K. 1969. Chromosome numbers in Zingiberaceae. Cytologia. 34(2):213-221.

Sato D. 1948. The karyotypes and phylogeny in Zingiberales. Japanese Journal of Genetics. 23:44-45.

Sato D. 1960. The karyotype analysis in Zingiberales with special reference to the protokaryotype and stable karyotype. Scientific Papers of the College of General Education, University of Tokyo. 10:225-243.

Sharma AK, Bhattacharyya NK. 1959. Cytology of several members of Zingiberaceae. Cellule. 50:297-346.

Simmonds NW. 1954. Chromosome behaviour in some tropical plants. Heredity. 8:139-146.

Specht CD. 2006. Systematics and evolution of the tropical monocot family Costaceae (Zingiberales): a multiple dataset approach. Systematic Botany. 31(1):89-106.

Specht CD, Stevenson DW. 2006. A new phylogeny-based generic classification of Costaceae (Zingiberales). Taxon. 55(1):153-163.

Stebbins GL. 1971. Chromosomal Evolution in Higher Plants. London: Addison-Wesley.

Subrahmanyam GV, Khoshoo TN. 1986. Cytological studies in the genus Costus L. Cytologia. 51:737-748.

Venkatasubban KR. 1946. A preliminary survey of chromosome numbers in Scitamineae of Bentham and Hooker. Proceedings of the Indian Academy of Sciences, Section B. 23(6):281-300.

Vovides AP, Lascurain M. 1995. Numeros cromosomicos de cuatro especies de Costus (Costaceae), una de Calathea, una de Maranta y una de Stromanthe (Marantaceae). Acta Botanica Mexicana. 33:81-86.

Weiss-Schneeweiss H, Emadzade K, Jang T-S, Schneeweiss GM. 2013. Evolutionary consequences, constraints and potential of polyploidy in plants. Cytogenetic and Genome Research. 140(2-4):137-150. 